\title{
THE ORIGIN OF TYPE I SPICULE OSCILLATIONS
}

\author{
D. B. Jess ${ }^{1}$, D. J. Pascoe ${ }^{2}$, D. J. Christian ${ }^{3}$, M. Mathioudakis ${ }^{1}$, P. H. Keys ${ }^{1}$, and F. P. Keenan ${ }^{1}$ \\ ${ }^{1}$ Astrophysics Research Centre, School of Mathematics and Physics, Queen’s University Belfast, Belfast, BT7 1NN, UK; d.jess@ qub.ac.uk \\ ${ }^{2}$ School of Mathematics and Statistics, University of St Andrews, St Andrews, KY16 9SS, UK \\ ${ }^{3}$ Department of Physics and Astronomy, California State University Northridge, Northridge, CA 91330, USA \\ Received 2011 October 15; accepted 2011 November 21; published 2011 December 8
}

\begin{abstract}
We use images of high spatial and temporal resolution, obtained with the Rapid Oscillations in the Solar Atmosphere instrument at the Dunn Solar Telescope, to reveal how the generation of transverse waves in Type I spicules is a direct result of longitudinal oscillations occurring in the photosphere. Here we show how pressure oscillations, with periodicities in the range of 130-440 s, manifest in small-scale photospheric magnetic bright points, and generate kink waves in the Sun's outer atmosphere with transverse velocities approaching the local sound speed. Through comparison of our observations with advanced two-dimensional magnetohydrodynamic simulations, we provide evidence for how magnetoacoustic oscillations, generated at the solar surface, funnel upward along Type I spicule structures, before undergoing longitudinal-to-transverse mode conversion into waves at twice the initial driving frequency. The resulting kink modes are visible in chromospheric plasma, with periodicities of 65-220 s, and amplitudes often exceeding $400 \mathrm{~km}$. A sausage mode oscillation also arises as a consequence of the photospheric driver, which is visible in both simulated and observational time series. We conclude that the mode conversion and period modification is a direct consequence of the $90^{\circ}$ phase shift encompassing opposite sides of the photospheric driver. The chromospheric energy flux of these waves are estimated to be $\approx 3 \times 10^{5} \mathrm{~W} \mathrm{~m}^{-2}$, which indicates that they are sufficiently energetic to accelerate the solar wind and heat the localized corona to its multi-million degree temperatures.
\end{abstract}

Key words: magnetohydrodynamics (MHD) - methods: numerical - Sun: atmosphere - Sun: chromosphere Sun: oscillations - Sun: photosphere

Online-only material: animations, color figures

\section{INTRODUCTION}

The origin of the energy required to power the solar wind, and heat the Sun's corona to its multi-million degree temperatures, remains an elusive mystery in physics. One promising mechanism involves the creation of Alfvénic waves near the solar surface, which can penetrate upward through the Sun's atmosphere with minimal reflection or energy loss (Erdélyi \& Fedun 2007). To date, there has been great controversy surrounding the interpretation of periodic transverse motions as Alfvén waves. Magnetohydrodynamic (MHD) waves with a wave number, $m=1$, can be considered "kink" oscillations when structured by a cylindrical waveguide. The associated periodic transverse motions imply a degree of plasma non-uniformity, resulting in intrinsic MHD waves exhibiting mixed (i.e., slow, fast, Alfvén) properties depending on the local plasma parameters. As a result, the term "Alfvénic" was introduced to describe waves which are predominantly characterized by the signatures displayed by pure Alfvén waves. Accordingly, transverse kink oscillations may often be considered Alfvénic in nature, providing they are incompressible, exhibit no intensity fluctuations along the structure, and display periodic displacements with the magnetic tension as the restoring force (Goossens et al. 2009). Thus, by definition, Alfvénic waves require strong magnetic field concentrations, and/or a steep density gradient with the external plasma, to act as waveguides (Van Doorsselaere et al. 2008), allowing energy to be directly channeled through the solar atmosphere. In the Sun's atmosphere, magnetic field lines clump together into tight bundles, forming flux tubes. The ubiquitous nature of Alfvénic waves in magnetic flux tubes has been demonstrated in a range of chromospheric and coronal plasmas (De Pontieu et al. 2007a; Tomczyk et al. 2007; Banerjee et al. 2009; Jess et al. 2009).
Spicules are dynamic, straw-like magnetic structures found in the solar chromosphere, and can be divided into two distinct classes (Zaqarashvili \& Erdélyi 2009). We will focus on Type I spicules, which are ubiquitous throughout the solar atmosphere, and live longer $(\approx 10$ minutes) than their jet-like Type II counterparts (De Pontieu et al. 2007b). The narrow shape of all spicule structures $(<600 \mathrm{~km})$, coupled with their rapidly evolving characteristics, places an utmost requirement for both high spatial and temporal resolution instrumentation. Recently, De Pontieu et al. (2007a) and He et al. (2009) observed Type I spicules, which displayed periodic transverse motions with periods ranging from 50-500 s, coupled with velocity amplitudes exceeding $25 \mathrm{~km} \mathrm{~s}^{-1}$. Unfortunately, such image sequences of spicules have been tied to limb observations. While observations at the solar limb allow spicule height information to be easily retrieved, they do not allow simultaneous measurements of the Sun's photosphere to be studied due to inherent line-of-sight effects. However, De Pontieu et al. (2011) and McIntosh et al. (2011) have recently shown how spicule dynamics make them ideal candidates in the quest for discovering efficient energy propagation into higher layers of the Sun's atmosphere. While transverse kink motion has been shown to be abundant in spicule observations, the underlying cause of the periodic motions has remained speculative. Overshooting of convective motions in the photosphere, granular buffeting, rebound shocks, and global $p$-mode oscillations have all been suggested as candidates for the creation of spicule oscillations (Roberts 1979; Sterling \& Hollweg 1988; Vranjes et al. 2008). It is known that oscillations in the lower solar atmosphere are prone to aspects of mode coupling, whereby one particular magnetoacoustic wave mode (normally classified as "fast" or "slow"; Nakariakov \& Verwichte 2005) may couple, and hence transfer wave energy, 
into another mode when atmospheric conditions are suitable (Ulmschneider et al. 1991; Kalkofen 1997). The rapid drop in density above the solar surface, coupled with the close proximity to oscillatory drivers, makes the photosphere and chromosphere an ideal location for the occurrence of efficient mode coupling. Indeed, McAteer et al. (2003) uncovered upward-propagating transverse oscillations, generated in large $(>2000 \mathrm{~km}$ diameter) photospheric bright points, which coupled into longitudinal waves at chromospheric heights. Often, the coupled oscillations demonstrate power at frequencies equal to twice the original, as a result of nonlinear interactions (Ulmschneider et al. 1991). Contrarily, it has been demonstrated, both analytically and theoretically, that when the magnetic pressure is approximately equal to the gas pressure (plasma $\beta=1$ ) in a solar flux tube, longitudinal-to-transverse mode coupling may also occur (De Moortel et al. 2004). To date, however, this form of "reversed mode coupling" has not been verified observationally. In this Letter, we undertake a multi-wavelength approach, allowing Type I spicules to be traced back to their anchor points on the solar surface. We provide conclusive evidence on how these ubiquitous oscillations manifest, and suggest what role they may take in the localized heating of the solar corona.

\section{OBSERVATIONS}

The data presented here are part of an observing sequence obtained during 13:46-14:47 UT on 2009 May 28, with the Richard B. Dunn Solar Telescope (DST) at Sacramento Peak, New Mexico. The newly commissioned Rapid Oscillations in the Solar Atmosphere (ROSA; Jess et al. 2010b) six-camera system was employed to image a $69.3 \times 69^{\prime \prime} .1$ region positioned at solar disk center. A spatial sampling of 0.'069 per pixel was used for the ROSA cameras to match the telescope's diffractionlimited resolution in the blue continuum to that of the CCD. This results in images obtained at longer wavelengths being slightly oversampled. However, this was deemed desirable to keep the dimensions of the field of view the same for all ROSA cameras.

During the observations presented here, high-order adaptive optics (Rimmele 2004) were used to correct wavefront deformations in real time. The acquired images were further improved through speckle reconstruction algorithms (Wöger et al. 2008), utilizing $16 \rightarrow 1$ restorations, providing reconstructed cadences of $0.528 \mathrm{~s}$ ( $\mathrm{G}$ band) and $4.224 \mathrm{~s}$ ( $\mathrm{Ca}$ II $\mathrm{K}$ and $\mathrm{H} \alpha$ ). More details of our instrumentation setup can be found in Jess et al. (2010a). Atmospheric seeing conditions remained excellent throughout the time series. However, to insure accurate co-alignment in all bandpasses, broadband time series were Fourier co-registered and de-stretched using a $40 \times 40$ grid, equating to a $\approx 1^{\prime \prime} .7$ separation between spatial samples (Jess et al. 2007, 2008). Narrowband images were corrected by applying de-stretching vectors established from simultaneous broadband reference images (Reardon et al. 2008).

\section{ANALYSIS AND DISCUSSION}

The ROSA field of view shows a range of features, including exploding granules and a multitude of magnetic bright points (MBPs; Figure 1). A large conglomeration of MBPs were located at heliocentric coordinates $\left(4^{\prime \prime},-3^{\prime \prime}\right)$, or S01W00 in the solar north-south-east-west coordinate system, providing an opportunity to examine these kilogauss structures without the line-of-sight spatial offsets normally associated with nearlimb observations. Examination of $\mathrm{H} \alpha$ images reveals a wealth of chromospheric jets and spicules, anchored into the solar
Table 1

Spicules Observed in Both Ca II K and $\mathrm{H} \alpha$ Data Sets

\begin{tabular}{lccccc}
\hline \hline $\begin{array}{l}\text { Spicule } \\
\text { Number }\end{array}$ & $\begin{array}{c}\text { Spicule } \\
\text { Length } \\
(\mathrm{km})\end{array}$ & $\begin{array}{c}\text { Kink } \\
\text { Period } \\
(\mathrm{s})\end{array}$ & $\begin{array}{c}\text { Kink } \\
\text { Amplitude } \\
(\mathrm{km})\end{array}$ & $\begin{array}{c}\text { Peak Kink } \\
\text { Velocity } \\
\left(\mathrm{km} \mathrm{s}^{-1}\right)\end{array}$ & $\begin{array}{c}\text { MBP Anchor } \\
\text { Location }\end{array}$ \\
\hline 1 & 4700 & 220 & 670 & 19.2 & $\left(8^{\prime \prime},-1^{\prime \prime}\right)$ \\
2 & 5200 & 139 & 630 & 28.3 & $\left(0^{\prime \prime},-2^{\prime \prime}\right)$ \\
3 & 3900 & 65 & 160 & 14.8 & $\left(1^{\prime \prime},-2^{\prime \prime}\right)$ \\
4 & 4100 & 158 & 410 & 16.2 & $\left(3^{\prime \prime},-4^{\prime \prime}\right)$ \\
$5^{\mathrm{a}}$ & 6200 & 129 & 380 & 18.5 & $\left(3^{\prime \prime},-2^{\prime \prime}\right)$ \\
6 & 4800 & 105 & 200 & 11.8 & $\left(8^{\prime \prime},-4^{\prime \prime}\right)$ \\
$7^{\mathrm{b}}$ & 5100 & 171 & 190 & 7.2 & $\left(8^{\prime \prime},-5^{\prime \prime}\right)$ \\
\hline
\end{tabular}

Notes.

a Spicule used for display purposes in Figures 1-3.

b Spicule used for display purposes in Figure 4.

surface at the locations of MBPs. These data reveal how unanchored ends of magnetic flux tubes are observed to sway periodically over many complete cycles (supplementary movies 1 and 2). To quantify the oscillatory parameters, a series of onedimensional slits, each separated by $1^{\prime \prime}(725 \mathrm{~km})$, were placed perpendicular to the Type I spicules along their entire length (upper panels of Figure 1). The resulting time-distance cuts reveal numerous periodic transversal displacements of these chromospheric structures as a function of time. The sinusoid fitting algorithms of Ballai et al. (2011) were used to extract periodic signatures from the transversal displacements present in the time-distance plots. We concentrate on the seven most prominent Type I spicules, observed in both $\mathrm{Ca}$ II $\mathrm{K}$ and $\mathrm{H} \alpha$ time series, which can be accurately traced back to their photospheric counterparts. The lower panel of Figure 1 displays a sample time-distance cut, revealing how these spicules demonstrate transverse kink oscillations above their corresponding MBP in the period range 65-220 s, with displacement amplitudes reaching as high as $670 \mathrm{~km}$. Velocity amplitudes often exceed $15 \mathrm{~km} \mathrm{~s}^{-1}$, indicating that these dynamic motions are close to the chromospheric sound speed (Fossum \& Carlsson 2005). Table 1 displays key characteristics of the spicules under investigation.

No periodic transverse motions in either the MBP edge, or bright central peak, were detected in simultaneous photospheric G-band images, implying that either spicule motion is generated independent of processes manifesting on the solar surface, or that a form of efficient mode conversion between the two layers (photosphere and chromosphere) is occurring. To investigate the latter, G-band intensities were averaged over the entire MBP structure under investigation, with fast Fourier transforms and wavelet analysis routines subsequently applied to the resulting intensity fluctuations. We find significant oscillatory power (exceeding 500 times the quiescent background) in the averaged intensity of these G-band features, which typically occupy $\sim 50$ pixels, or $130,000 \mathrm{~km}^{2}$. Furthermore, the most powerful intensity oscillations found in each photospheric MBP are at twice the period (130-440 s) of the corresponding chromospheric transverse oscillation, indicating the presence of frequency doubling. The dominant period remains constant over each MBP surface, indicating the presence of powerful, yet coherent, drivers. We further utilize our high-resolution observations to deduce additional parameters related to the longitudinal photospheric drivers, and find that the peak longitudinal oscillatory power is located at the center of each photospheric MBP, with a magnitude exceeding $7 \times 10^{5}$ times the background average (right panel of Figure 2). Power decreases radially away 

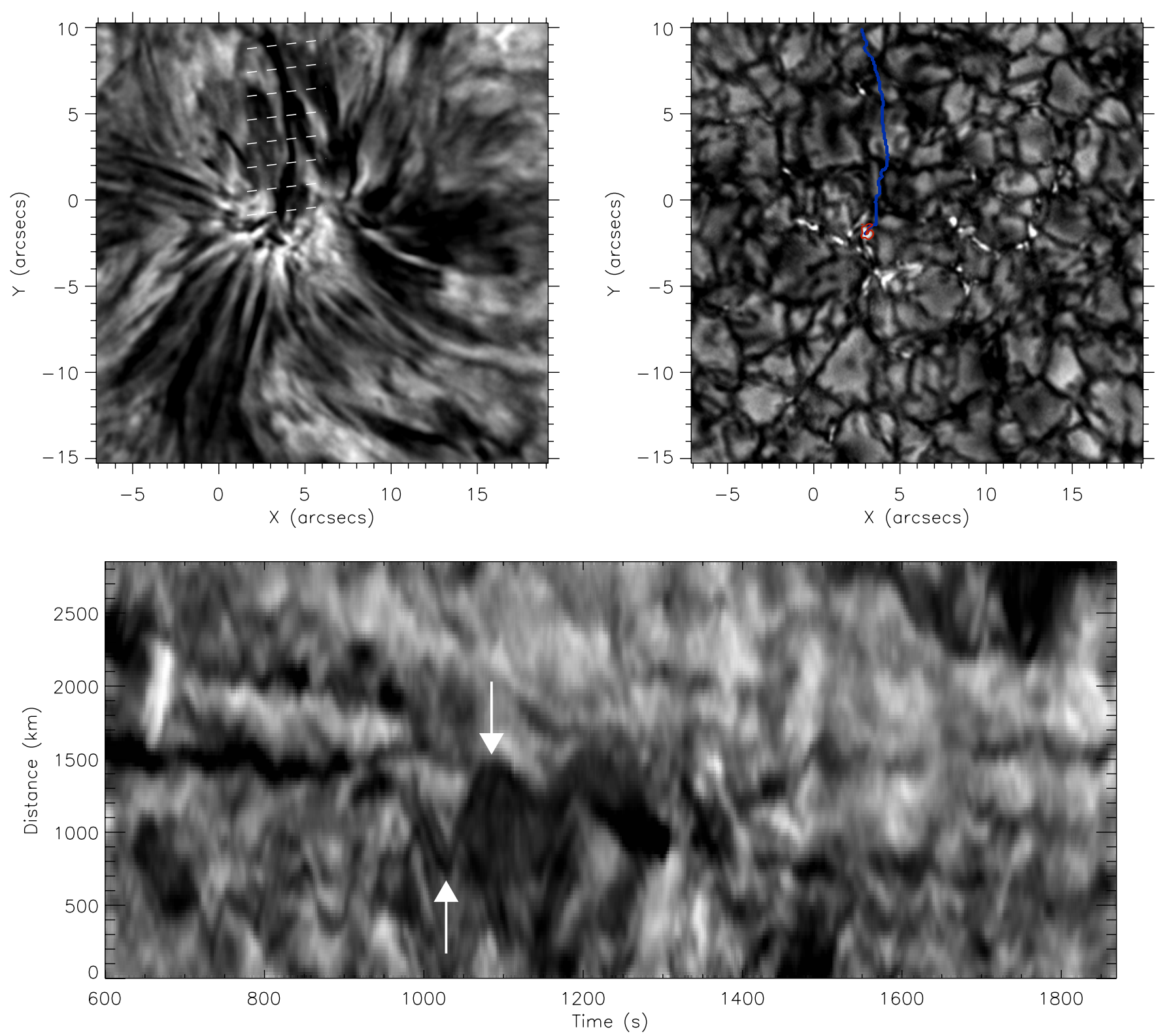

Figure 1. Simultaneous images of the $\mathrm{H} \alpha$ core (chromosphere; upper left) and $\mathrm{G}$ band (photosphere; upper right), acquired at 14:06:21 UT on 2009 May 28. Dashed white lines in the $\mathrm{H} \alpha$ core image highlight the spatial positions where time-distance cuts are made. The blue trace in the G-band image denotes the position of a chromospheric spicule (visible in the left panel as a dark, straw-like structure), where one end is anchored into the photosphere above an MBP. A red contour indicates the location where a high concentration of longitudinal oscillatory power is present. The lower panel is a sample $\mathrm{H} \alpha$ time-distance cut, obtained $4000 \mathrm{~km}\left(5^{\prime \prime} 5\right)$ from the underlying MBP, revealing an abundance of periodic transverse motions in the solar chromosphere (see also supplementary movies 1 and 2). White arrows highlight a trough and a peak of a typical transverse oscillation. The scale is in heliocentric coordinates, where $1^{\prime \prime}$ approximately equals $725 \mathrm{~km}$.

(Animations (Movies 1 and 2) and a color version of this figure are available in the online journal.)

from the center of the photospheric MBP, eventually dropping to zero at the edge of the structure. We compute the spatial distributions of the oscillatory phase for the photospheric MBPs relative to this position of peak power. Figure 2 reveals how deviations in phase angle, from the positions of peak power, are within the range of $+40^{\circ}$ to $-49^{\circ}$, providing absolute phase shifts of $\approx 90^{\circ}$ across each entire MBP structure. These phase shifts remain constant throughout the duration of periodic behavior, with oscillatory power eventually ceasing as the MBP fragments. In addition, the detected photospheric MBP periodicities (130-440 s) overlap with the solar $p$-mode spectrum (Leighton et al. 1962; Ulrich 1970), suggesting these global modes provide the underlying driving force required to generate spicule oscillations.
To investigate the temporal variations in wave behavior, we calculate the oscillatory power at each time step, and compare with simultaneous measurements of the same spicule observed in different wavelengths. Our analysis shows that the power of lower chromospheric (Ca II $\mathrm{K}$ ) transverse oscillations peaks after the photospheric (G-band) longitudinal periodicities, yet before the upper chromospheric $(\mathrm{H} \alpha)$ oscillations. Figure 3 reveals how a discernible time lag exists between three distinct layers of the lower solar atmosphere. This implies that the observed chromospheric spicule oscillations are a result of upwardly propagating magnetoacoustic wave modes, generated at the solar surface, which couple into transverse kink waves in a region encompassing the photospheric/chromospheric boundary. An important trend to consider is how the power of 

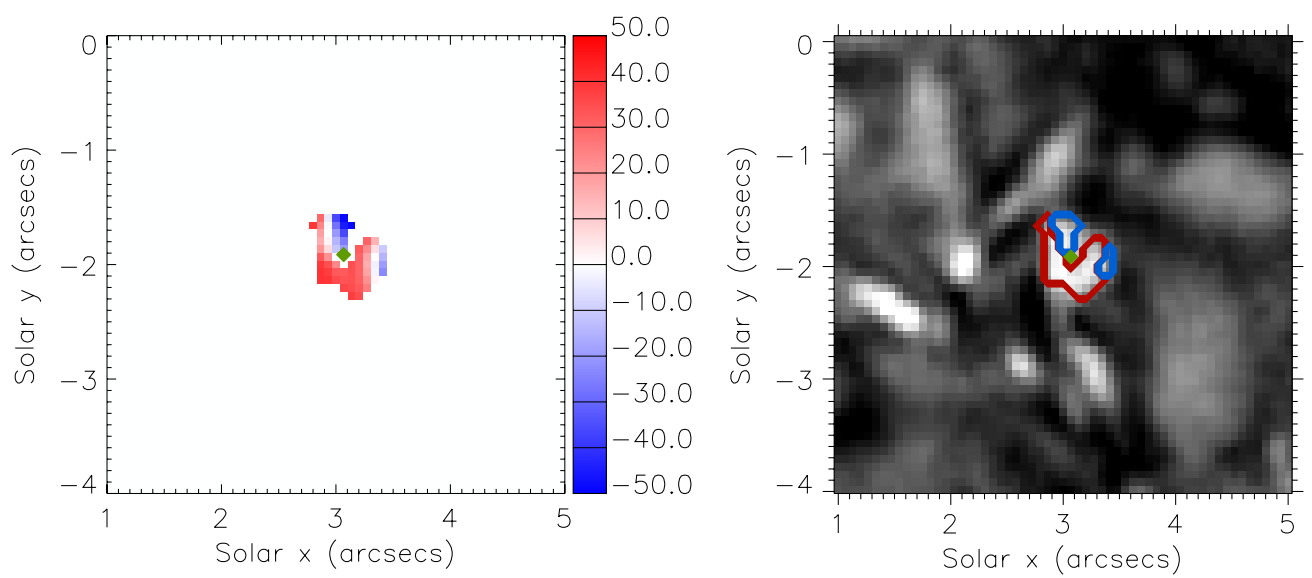

Figure 2. Zoom-in of an MBP group acting as the photospheric anchor point of a chromospheric spicule. Maximum longitudinal oscillatory power is found at the center of the MBP (green pixel; left panel), equating to power in excess of $7 \times 10^{5}$ times the quiescent background. Phase shifts (in degrees) relative to this local maximum are displayed using the red/blue color table, with maximum values in this example of $+40^{\circ}$ and $-49^{\circ}$. The right panel displays the phase information contoured on top of the simultaneous G-band intensity image, revealing how oscillatory power is closely confined within the MBP perimeter.

(A color version of this figure is available in the online journal.)

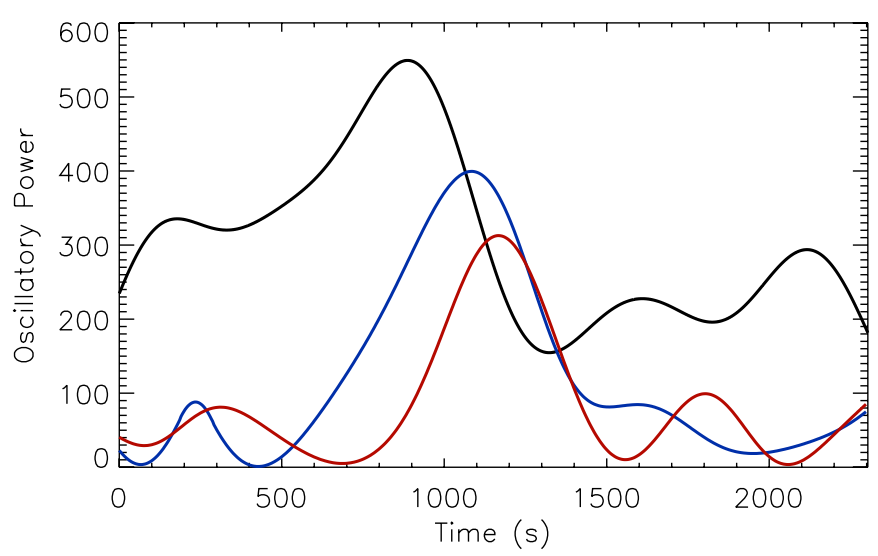

Figure 3. Oscillatory power of an isolated MBP group in the $\mathrm{G}$ band (black), and associated $\mathrm{Ca}$ II $\mathrm{K}$ (blue), and $\mathrm{H} \alpha$ (red) spicule time series. The $x$-axis is time from the start of the observing sequence, while the $y$-axis is power averaged over the entire MBP/spicule structure, and normalized to the background quiescent Sun. G-band power is for oscillations in intensity at a period of $258 \mathrm{~s}$, while the $\mathrm{Ca}$ II $\mathrm{K}$ and $\mathrm{H} \alpha$ power is derived from the periodic transverse displacements of the spicule with a periodicity of 129 s. It is clear the G-band power leads the $\mathrm{Ca}$ II $\mathrm{K}$ and $\mathrm{H} \alpha$ time series, indicating the presence of an upward propagating magnetoacoustic wave, which couples into an transverse kink mode near the chromospheric boundary.

(A color version of this figure is available in the online journal.)

detected oscillatory behavior varies as a function of atmospheric height. Power spectra for the G-band intensity, and Ca II K and $\mathrm{H} \alpha$ transverse oscillations, were normalized to their respective quiescent backgrounds before comparison. Figure 3 shows an appreciable decrease in oscillatory power as a function of atmospheric height. This has important consequences, as it suggests the presence of physical damping, and therefore conversion into heat, of the observed wave modes. Further examination of each MBP reveals multiple power peaks (also visible in Figure 3), suggesting that the creation, and subsequent transverse behavior of solar spicules, is quasi-periodic in nature. This is the first observational evidence of longitudinal-to-transverse mode coupling occurring in the Sun's atmosphere.

To further investigate the propagation and coupling of these wave phenomena, we utilized advanced two-dimensional MHD simulations. These simulations were performed using the Lare2D code (Arber et al. 2001), where a spicule was modeled as a density enhancement, embedded in a straight, uniform magnetic field. The spicule density has a symmetric Epstein profile (Nakariakov \& Roberts 1995) in the transverse direction, with a peak density 100 times the background value, and a diameter of approximately $600 \mathrm{~km}$ (upper-left panel of Figure 4). The magnetic field strength is chosen to give an external Alfvén speed of $362 \mathrm{~km} \mathrm{~s}^{-1}$, and an Alfvén speed of $36.2 \mathrm{~km} \mathrm{~s}^{-1}$ at the center of the spicule. A temperature profile is chosen to give a plasma $\beta=1$, where efficient mode coupling is expected to occur (De Moortel et al. 2004). The simulation has a resolution of $600 \times 2000$ grid points, providing a numerical domain size of $4000 \times 20,000 \mathrm{~km}^{2}$. For the purposes of this investigation, only effects up to a few thousand kilometers (i.e., a region incorporating the photosphere and chromosphere) will be considered.

An artificial driver is applied at the lower boundary in the form of a longitudinal velocity component. This is consistent with the observational interpretation that the driver arises from a global $p$-mode oscillation. The coherent driver is applied across the diameter of the spicule, with a periodicity of $215 \mathrm{~s}$, and a maximum amplitude of $12.5 \mathrm{~km} \mathrm{~s}^{-1}$. However, to remain consistent with parameters derived from the observational time series, one side of the simulated MBP has a phase difference of $90^{\circ}$ with respect to the other (upper-left panel of Figure 4). As the driver is longitudinal and compressive, a $90^{\circ}$ phase shift creates transverse (i.e., across the spicule) gradients in pressure which displace the spicule axis, generating the kink mode, in addition to producing periodic compressions and rarefactions (sausage mode). These periodic pressure differences, across the body of the spicule, also induce a frequency doubling of the coupled transverse wave, visible in the upper-right panel of Figure 4 by a change from a purely sinusoidal waveform. Both transverse kink and sausage modes are readily apparent in the lower panels of Figure 4, where time-distance cuts of observational and simulated data are displayed (see also supplementary movies 3 and 4). In our simulations, the magnitude of the excited kink mode is smaller than the amplitude of the input longitudinal driver. This is a consequence of the uniform magnetic field strength used. In the real solar atmosphere, the magnetic field strength decreases rapidly with height, which is a likely contributor to the more prominent increase in kink amplitude seen in current ground- and space-based observations of chromospheric spicules (e.g., He et al. 2009). 

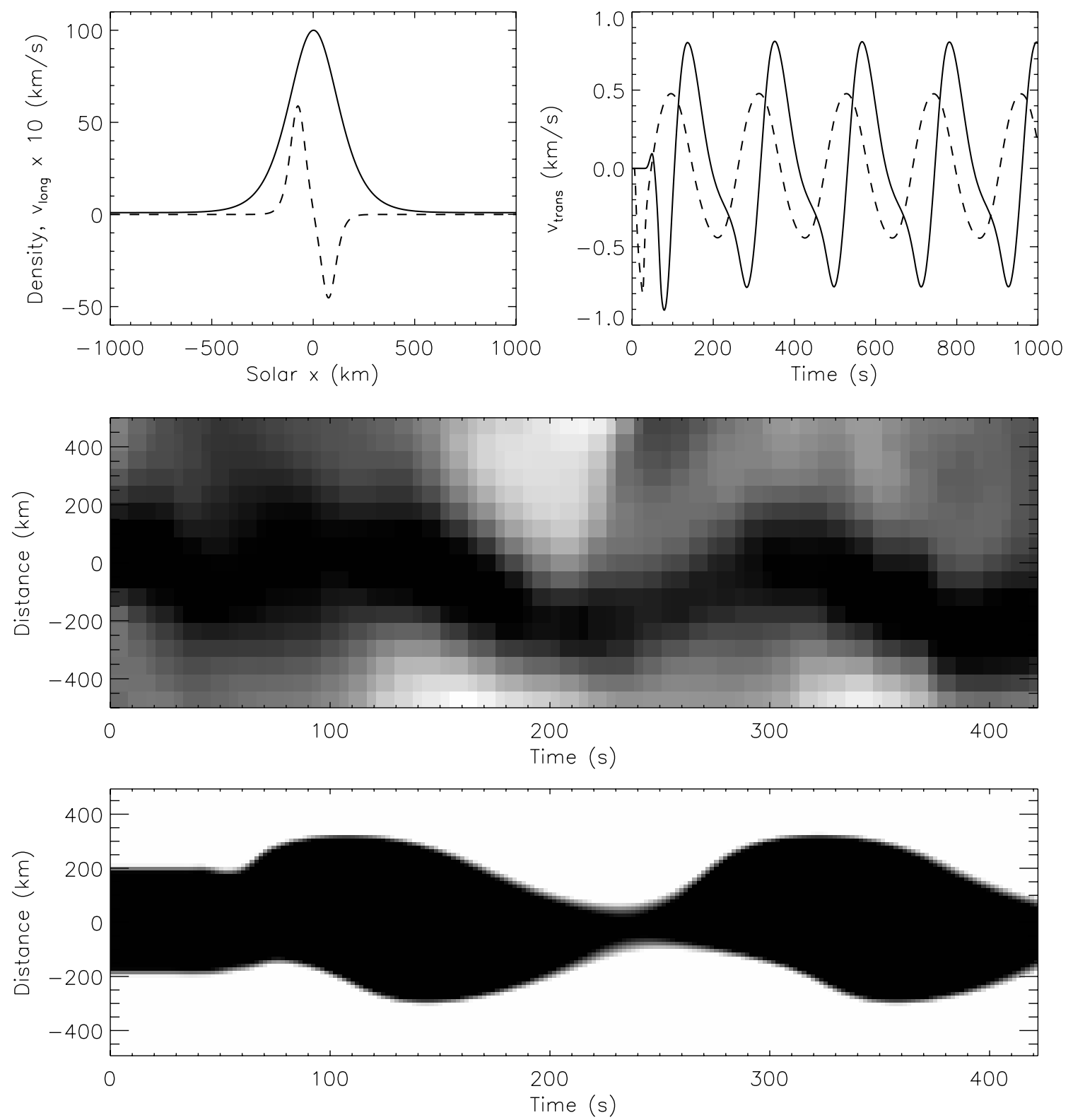

Figure 4. Upper left: the spicule density profile (solid line) normalized to the background value, while the dashed line shows a snapshot of the longitudinal velocity amplitude, $130 \mathrm{~s}$ into the simulation, and scaled for clarity. Upper right: the transverse velocity signal, measured at the spicule axis, as a function of time for two heights. The dashed and solid lines represent the signal obtained at heights of $500 \mathrm{~km}$ and $2000 \mathrm{~km}$ above the photosphere, respectively. A periodic transverse velocity signal, which increases in amplitude away from the photosphere, reveals the progression of longitudinal-to-transverse mode coupling. Frequency doubling, represented by a change from a purely sinusoidal waveform, is observed in the signal from a height of $2000 \mathrm{~km}$. Middle: a time-distance cut of a chromospheric spicule observed in $\mathrm{H} \alpha$. Bottom: a time-distance cut of the simulated spicule density, at a height of $2000 \mathrm{~km}$ above the photospheric boundary. There is a remarkable degree of similarity between the two lower panels, with both kink (transverse displacement of spicule axis) and sausage (periodic compression and rarefaction) modes visible. Supplementary movies 3 and 4 display time-lapse image sequences of the simulations presented here.

(Animations (Movies 3 and 4) of this figure are available in the online journal.)

Nevertheless, the upper-right panel of Figure 4 indicates that the longitudinal-to-transverse mode-coupling mechanism present in our MHD simulations does promote progressive increases in kink amplitude as a function of atmospheric height.

By combining observed photospheric periodicities and phase distributions, in conjunction with realistic MBP structuring, our simulations reveal efficient, and ubiquitous, mode conversion of longitudinal oscillations into their transverse kink counterparts, at twice the driven frequency. The simulations display wave properties consistent with our observations, and demonstrate how transverse spicule oscillations, with significant amplitudes, readily exist in the solar atmosphere, and therefore have important consequences for energy transportation into the solar corona. We estimate the energy flux, $E$, of the observed chromospheric waves using (De Pontieu et al. 2007a)

$$
E=\rho v^{2} v_{A},
$$

where $\rho$ is the mass density of the flux tube, $v$ is the observed velocity amplitude, and $v_{A}$ is the corresponding Alfvén speed, defined as $v_{A}=B / \sqrt{\mu}_{0} \rho$, with $\mu_{0}$ the magnetic permeability. For a mass density of $\rho \approx 1.3 \times 10^{-8} \mathrm{~kg} \mathrm{~m}^{-3}$, and a magnetic field strength $B \approx 12 \mathrm{G}$, derived from a bright network chromospheric model (Vernazza et al. 1981), coupled with an observed velocity amplitude of $v \approx 15 \mathrm{~km} \mathrm{~s}^{-1}$, the energy flux in the chromosphere is $E \approx 3 \times 10^{5} \mathrm{~W} \mathrm{~m}^{-2}$. It is estimated 
that approximately $2.2 \%$ of the solar surface is covered by MBPs (Sánchez Almeida et al. 2010), and if each MBP is linked to a single corresponding chromospheric Type I spicule, it equates to an average global energy of $660 \mathrm{~W} \mathrm{~m}^{-2}$. Current work suggests waves with an energy flux $\approx 100 \mathrm{~W} \mathrm{~m}^{-2}$ are sufficiently vigorous to heat the localized corona and launch the solar wind when their energy is thermalized (Jess et al. 2009). Therefore, a transmission coefficient of $\approx 15 \%$ through the thin transition region would provide sufficient energy to heat the entire corona. Regions on the solar surface containing highly magnetic structures, such as sunspots, pores, and large MBP groups, should possess even higher mass densities and magnetic field strengths, in addition to a greater numbers of spicules. In this regime, the energy flux available to heat the corona will be significantly higher than the minimum value required to sustain localized heating.

\section{CONCLUDING REMARKS}

We have utilized images of high spatial and temporal resolution, obtained with the ROSA instrument at the DST, to reveal how the generation of transverse kink oscillations in Type I spicules is a direct result of mode conversion in the lower solar atmosphere. Through comparison of our observations with advanced two-dimensional MHD simulations, we show how longitudinal pressure modes in photospheric MBPs, with periodicities in the range 130-440 s, funnel upward through the Sun's atmosphere, before converting into kink modes at twice the initial frequency, often with amplitudes exceeding $400 \mathrm{~km}$. We conclude that the mode conversion and period modification is a direct consequence of the $90^{\circ}$ phase shift encompassing opposite sides of the photospheric driver. This is the first observational evidence of this mechanism occurring in the solar atmosphere. Energy flux estimates for these oscillations indicate that the waves are sufficiently energetic to accelerate the solar wind and heat the quiet corona to its multi-million degree temperatures.

The naming of transverse oscillations observed in solar structures remains highly controversial, with definitions revolving around "Alfvén," "Alfvénic," and "magnetosonic kink" terminology. While our observations demonstrate signatures consistent with previous studies on Alfvénic waves (e.g., De Pontieu et al. 2007a; McIntosh et al. 2011), we have deliberately chosen to describe the observed periodic motions simply as transverse kink waves. This is currently the most unopposed description of such wave phenomena, and avoids the potential pitfalls of what is a rapidly developing area within solar physics.
D.B.J. thanks STFC for the award of a Post-Doctoral Fellowship. D.J.C. thanks CSUN for start-up funding related to this project. P.H.K. is grateful to NIDEL for a PhD studentship. Solar Physics research at QUB is supported by STFC. The ROSA project is supported by EOARD.

Facility: Dunn (ROSA)

\section{REFERENCES}

Arber, T. D., Longbottom, A. W., Gerrard, C. L., \& Milne, A. M. 2001, J. Comput. Phys., 171, 151

Ballai, I., Jess, D. B., \& Douglas, M. 2011, A\&A, 534, A13

Banerjee, D., Pérez-Suárez, D., \& Doyle, J. G. 2009, A\&A, 501, L15

De Moortel, I., Hood, A. W., Gerrard, C. L., \& Brooks, S. J. 2004, A\&A, 425, 741

De Pontieu, B., McIntosh, S. W., Carlsson, M., et al. 2007a, Science, 318, 1574

De Pontieu, B., McIntosh, S. W., Carlsson, M., et al. 2011, Science, 331, 55

De Pontieu, B., McIntosh, S. W., Hansteen, V. H., et al. 2007b, PASJ, 59, 655

Erdélyi, R., \& Fedun, V. 2007, Science, 318, 1572

Fossum, A., \& Carlsson, M. 2005, Nature, 435, 919

Goossens, M., Terradas, J., Andries, J., Arregui, I., \& Ballester, J. L. 2009, A\&A, 503,213

He, J.-S., Tu, C.-Y., Marsch, E., et al. 2009, A\&A, 497, 525

Jess, D. B., Andić, A., Mathioudakis, M., Bloomfield, D. S., \& Keenan, F. P. 2007, A\&A, 473, 943

Jess, D. B., Mathioudakis, M., Christian, D. J., Crockett, P. J., \& Keenan, F. P. 2010a, ApJ, 719, L134

Jess, D. B., Mathioudakis, M., Christian, D. J., et al. 2010b, Sol. Phys., 261, 363

Jess, D. B., Mathioudakis, M., Crockett, P. J., \& Keenan, F. P. 2008, ApJ, 688, L119

Jess, D. B., Mathioudakis, M., Erdélyi, R., et al. 2009, Science, 323, 1582

Kalkofen, W. 1997, ApJ, 486, L145

Leighton, R. B., Noyes, R. W., \& Simon, G. W. 1962, ApJ, 135, 474

McAteer, R. T. J., Gallagher, P. T., Williams, D. R., et al. 2003, ApJ, 587, 806

McIntosh, S. W., de Pontieu, B., Carlsson, M., et al. 2011, Nature, 475, 477

Nakariakov, V. M., \& Roberts, B. 1995, Sol. Phys., 159, 399

Nakariakov, V. M., \& Verwichte, E. 2005, Living Rev. Sol. Phys., 2, 3

Reardon, K. P., Lepreti, F., Carbone, V., \& Vecchio, A. 2008, ApJ, 683, L207

Rimmele, T. R. 2004, Proc. SPIE, 5490, 34

Roberts, B. 1979, Sol. Phys., 61, 23

Sánchez Almeida, J., Bonet, J. A., Viticchié, B., \& Del Moro, D. 2010, ApJ, 715, L26

Sterling, A. C., \& Hollweg, J. V. 1988, ApJ, 327, 950

Tomczyk, S., McIntosh, S. W., Keil, S. L., et al. 2007, Science, 317, 1192

Ulmschneider, P., Zaehringer, K., \& Musielak, Z. E. 1991, A\&A, 241, 625

Ulrich, R. K. 1970, ApJ, 162, 993

Van Doorsselaere, T., Nakariakov, V. M., \& Verwichte, E. 2008, ApJ, 676, L73

Vernazza, J. E., Avrett, E. H., \& Loeser, R. 1981, ApJS, 45, 635

Vranjes, J., Poedts, S., Pandey, B. P., \& de Pontieu, B. 2008, A\&A, 478, 553

Wöger, F., von der Lühe, O., \& Reardon, K. 2008, A\&A, 488, 375

Zaqarashvili, T. V., \& Erdélyi, R. 2009, Space Sci. Rev., 149, 355 